\title{
Tyrosine Kinase Inhibitors in the Elderly: A Case Report and Literature Review on Toxicity Profiles
}

\author{
Aileen Deng, Andrew Chapman and Jianqing Lin
}

Division of Hematology/Oncology, Department of Urology, Co-Program Leader, Genitourinary Oncology Program, GW Cancer Center, George Washington University School of Medicine and Health Sciences, Pennsylvania, USA

Corresponding author: Jianqing Lin, Division of Hematology/Oncology, Department of Urology, Co-Program Leader, Genitourinary Oncology Program, GW Cancer Center, George Washington University School of Medicine and Health Sciences, 2150 Pennsylvania Ave, NW, Suite 1-208, Washington DC, 20037, USA, Tel: 202677 6851; Fax: 202-741-2487; E-mail: Jilin@mfa.gwu.edu

Rec date: March 20, 2016; Acc date: April 25, 2017; Pub date: April 28, 2017

Citation: Deng A, Chapman A, Lin J. Tyrosine Kinase Inhibitors in the Elderly: A Case Report and Literature Review on Toxicity Profiles. Arch Can Res. 2017, 5: 2.

\section{Abstract}

Tyrosine kinase inhibitors (TKIs) have emerged as the mainstay treatment for renal cell carcinoma (RCC). Despite growing popularity of TKIs, the treatment safety and tolerability in elderly patients are yet to be fully understood. As new assessment tools become available in identifying elderly patients with cancer at risk for severe treatment-related toxicities, much remains to be learned about how to integrate these tools into our management of elderly patients with cancer. We present a case of a 75year-old man with RCC who was initially treated with pazopanib and subsequently treated with sunitinib. We review the safety and toxicity profiles of TKIs in treating elderly patients with RCC.

Keywords: Geriatric oncology; Tyrosine kinase Inhibitors; Safety; Renal cell cancer

\section{Introduction}

The treatment of advanced renal cell carcinoma (RCC) has revolutionized over the past 10 years with the development of drugs that target the vascular endothelial growth factor (VEGF) and the mammalian target of rapamycin (mTOR) signaling axis. Tyrosine kinase inhibitors (TKIs), which target VEGF receptors and inhibit angiogenesis, have emerged as the mainstay treatment for RCC [1]. Despite the growing popularity of TKIs, available clinical trials with TKIs often underrepresent elderly patients. Thus, the treatment safety and tolerability in elderly patients are yet to be fully understood. In addition, tools to identify elderly patients most at-risk for severe treatment toxicities, while available, is currently not well integrated into our standard of care of elderly patients with cancer. We illustrate our current gaps in knowledge and review the current literature on safety profiles of TKIs in elderly patients with RCC by presenting a case of an elderly patient with RCC who was treated with TKIs.

\section{Case Report}

A 75-year-old man with a history of metastatic renal cell cancer was evaluated for unresponsiveness. His medical history is significant for hypertension and stage III chronic kidney disease. He has a 25-pack year smoking history and worked previously as an exterminator. An evaluation for microscopic hematuria revealed an $11.2 \times 14.7 \times 12.6 \mathrm{~cm}$ lobulated solid and cystic necrotic renal mass arising from the mid pole of the right kidney. Bilateral sub-centimeter lung nodules were present on initial staging CAT scan, concerning for metastatic disease. Given his good performance status, he underwent right radical nephrectomy. Surgical pathology confirmed RCC, clear cell type. The patient was then followed with serial imaging. Eighteen months later, MRI abdomen showed a new slow growing $1.3 \mathrm{~cm}$ lesion in the upper pole of the left kidney. The patient underwent CAT scan-guided biopsy followed by cryoablation of the left renal lesion. Surgical pathology confirmed RCC. Due to a progressive dry cough and enlarging pulmonary lesions, decision was made to start treatment for his metastatic RCC.

The patient was started on pazopanib at an initial dose of $600 \mathrm{mg}$ daily. Three weeks after starting pazopanib, he noticed progressive fatigue, nausea and decreased appetite. Due to worsening symptoms, he was brought to the emergency room. In the emergency room, the patient became unresponsive. Telemetry revealed 25 seconds of sinus arrest with ventricular asystole. EKG after the episode showed normal sinus rhythm with no evidence of ischemia and a QTc of $399 \mathrm{~ms}$. The patient emergently underwent transvenous pacemaker insertion. He had a second episode of unresponsiveness with 19 seconds of sinus arrest. His hospital stay was further complicated by thrombocytopenia and jaundice. His platelets decreased to 75 $B / L$ four days after sinus arrest. His liver function test revealed an elevated total bilirubin of 2.7, an elevated AST and ALT of 321 and 656 respectively and an elevated alkaline phosphatase of 656 eight days after sinus arrest. His symptoms and lab abnormalities improved and eventually resolved with supportive care.

Pazopanib was discontinued after this event. The patient was started on sunitinib at a reduced dose of $25 \mathrm{mg}$ daily. $\mathrm{He}$ 
tolerated sunitinib well, experiencing more energy and less nausea. While attempts were made to increase his sunitinib dose to $37.5 \mathrm{mg}$, he developed diarrhea, fatigue, oral stomatitis and hand-foot syndrome. The patient is currently maintained on sunitinib $25 \mathrm{mg}$ alternating with $37.5 \mathrm{mg}$ daily for two weeks on one week off schedule.

\section{Review of Literature}

Available clinical trials with TKIs in RCC often underrepresented elderly patients. In a retrospective, singlecenter analysis of 219 patients with metastatic RCC (mRCC), only $20(9.1 \%)$ patients were age 75 or older. The study found that patients age 75 years and older received fewer lines of systemic therapy as compared to other age-based subsets and more often discontinued treatments due to toxicity [2].

\section{Sorafenib}

Among the various TKIs, sorafenib and its tolerability in elderly patients appear to be the most-studied. Sorafenib was the first TKI approved for the treatment of metastatic RCC [3]. Adverse events appear to be similar in older patients when compared to younger patients at a starting dose of sorafenib $400 \mathrm{mg}$ twice daily. In a retrospective subgroup analysis of data from the Treatment Approach in Renal Cancer Global Evaluation Trial (TARGET), which included 115 patients 70 years and older, adverse events were predictable and manageable regardless of age [4]. The most common adverse events among older sorafenib-treated patients were rash, diarrhea, alopecia, fatigue, hand-foot syndrome and anorexia with a higher incidence of gastrointestinal symptoms in older patients compared to younger patients. While a higher incidence of grade 3 adverse events was reported in older patients treated with sorafenib, $79 \%$ of older patients tolerated treatment with sorafenib.

Similar results were echoed in other analysis $[5,6]$. No difference in efficacy or safety was found in patients older than 70 years compared to younger patients. A higher incidence of grade 3 or 4 adverse effects were seen in older patients, 75 years and older, compared to younger patients [6]. While the safety profile appears to be similar in older and younger patients, treatment duration appears to be shorter in older patients by $30 \%$. In practice, if a dose reduction is needed, sorafenib $400 \mathrm{mg}$ twice daily can be decreased to once daily or every other day [7].

\section{Pazopanib}

Pazopanib was approved in the US in 2009 [8]. There is still relatively little data on the safety profiles of pazopanib in elderly patients. In a randomized, double-blinded, placebocontrolled phase III trial on pazopanib in RCC, the most common adverse effects in the pazopanib group with a median age of 59 years included diarrhea, hypertension, hair discoloration, nausea, anorexia and vomiting [9]. The most common grade 3 or 4 adverse effects in the pazopanib group were AST and ALT elevation ( $7 \%$ and $10 \%$ respectively), hypertension (4\%) and diarrhea (4\%). The recommended dosage of pazopanib is $800 \mathrm{mg}$ daily, which can be dosereduced to $400 \mathrm{mg}$ daily to minimize toxicities [10].

\section{Sunitinib}

Sunitinib is typically started at $50 \mathrm{mg}$ daily in 6-week cycles [11,12]. It was approved in 2006 for the treatment of RCC [13]. The most common adverse events include fatigue and diarrhea. Other common adverse events include nausea, stomatitis, dyspepsia, hypertension and hand-foot syndrome. In an expanded-access trial looking at the safety and efficacy of sunitinib for metastatic RCC, the most common grade 3 or 4 adverse events did not differ in elderly patients [14]. High age has been highly correlated with severe toxicity with sunitinib [15]. In a study looking at 82 patients with advanced RCC on sunitinib dosed $50 \mathrm{mg}$ daily, 4 weeks on, 2 weeks off, $49 \%$ of patients experienced severe toxicity leading to dose reduction or drug discontinuation. The most common reasons for dose reduction were stomatitis, followed by fatigue and hand-foot syndrome. Severe toxicity highly correlated with low body surface area, high age and female gender.

\section{Axitinib}

Axitinib was approved in 2012 and is currently indicated for the treatment of RCC after failure of one systemic therapy. Similar to other TKIs, there is currently little data regarding the use in elderly patients. In a multicenter controlled trial comparing axitinib to sorafenib, the most common adverse events associated with axitinib were diarrhea, hypertension, fatigue, decreased appetite, nausea and dysphonia [16]. In unpublished data from Pfizer, patients 65 years and older treated with axitinib had higher incidences of fatigue, weight loss and decreased appetite compared to that in younger patients [17]. While grade 3 or greater adverse events were similar in older and younger patients on axitinib, older patients had a higher incidence of fatigue compared to that in younger patients. Axinitib is the only targeted agent that benefits from recommended titration [18]. Currently, the recommended oral starting dose of axitinib is $5 \mathrm{mg}$ twice a day [19]. In patients who do not develop dose-limiting toxicity, up-titration to $7 \mathrm{mg}$ twice a day two weeks after initiation is suggested. Further titration is recommended to a maximum of $10 \mathrm{mg}$ twice a day. Patients who develop adverse effects that require dose reduction should be decreased to $3 \mathrm{mg}$ twice a day or $2 \mathrm{mg}$ twice a day.

\section{Discussion}

Our patient was started on pazopanib $600 \mathrm{mg}$ daily. He developed fatigue, anorexia, nausea, and AST and ALT elevation some of the most common adverse effects associated with pazopanib. Sinus arrest is an extremely rare adverse effect of pazopanib. In clinical trials with pazopanib, cardiac dysfunction such as decreased left ventricular ejection fraction and congestive heart failure was only observed in $0.6 \%$ of patients [8]. In a randomized, double-blind study on the effect of repeated oral doses of pazopanib on cardiac conduction in patients with solid tumors, pazopanib produced 
a concentration-dependent decrease in heart rate [20]. While the cardiac toxicity associated with TKI is thought to be due to QTc prolongation [21], our patient had a normal QTc. After changing to sunitinib, our patient was only able to tolerate sunitinib at reduced doses and duration. He developed diarrhea, fatigue, oral stomatitis and hand-foot syndrome at higher doses of sunitinib, all common adverse effects of sunitinib.

Currently, two studies have compared pazopanib with sunitinib $[22,23]$. Patients on pazopanib tend to have a higher incidence of changes in hair color, weight loss and alopecia and less incidence of fatigue and hand-foot syndrome when compared to patients on sunitinib [23]. In addition, pazopanib appears to have a similar cardiovascular safety profile when compared to sunitinib. When looking at quality of life and patient preference, patients tend to favor pazopanib over sunitinib. In the COMPARZ trial, 11 of the 14 health-related Quality of life (QoL) domains showed a statistical difference favoring pazopanib [23]. In the PISCES trial, there was a significant patient preference in favor of pazopanib $(70 \%$ versus $22 \%, \mathrm{P}<0.001$ ) with the most common reason being less fatigue and better overall QoL when choosing pazopanib and less diarrhea when choosing sunitinib [22].

His case raises the question, are there currently tools that can be used to risk stratify elderly patients and to identify patients at high risk of treatment toxicities? While current research suggests similar efficacy and safety in older patients compared to younger patients on sorafenib, are these older patients representative of the "average" older patient?

Chronological age is traditionally used in clinical trials and the majority of clinical research as a major descriptor of aging. However, it is widely recognized that chronological age does not always capture functional age. The need to capture the heterogeneous physiologic and functional status of the older adult has led to the development of geriatric assessment [24]. Geriatric assessment identifies factors other than chronologic age to predict morbidity and mortality among older adults. It considers a person's functional status, comorbid medical conditions, psychological state, cognitive function, social support and nutritional status. While geriatric assessment tools have been developed to identify elderly patients at high risk for chemotherapy intolerance, there remain no studies on the use geriatric assessment in elderly patients prior to TKIs.

The Comprehensive Geriatric Assessment (CGA) considers a patient's functional status, comorbidities, medications, nutritional status, cognitive function, psychological status, and social support system [25]. Studies combining standard oncology workup with CGA found that poor nutrition and cognition predict inability to tolerate 4 cycles of chemotherapy and poor nutrition and frailty is associated with decreased survival [26]. While some recommend performing CGA on every older adult with cancer prior to starting chemotherapy, others have proposed the use of pre-screening tools that takes less time and can further identify those patients that should be evaluated using the full CGA [27-30].
Predictive tools that use clinical characteristics to categorize patients at risk for severe chemotherapy toxicity may also help identify at-risk elderly patients. The Cancer and Aging Research Group (CARG) toxicity tool help predict severe chemotherapy toxicity by looking at risk factors such as age 72 years and older, genituourinary or gastrointestinal primary site, hemoglobin, creatinine clearance, standard treatment dosing, multiple chemotherapy agents, and other CGA factors [31]. The Chemotherapy Risk Assessment Scale for High-Age Patients (CRASH) helps predict grade 3 or greater nonhematologic toxicity and grade 4 or greater hematologic toxicity [32].

More integration of geriatric assessment or predictive tools in current clinical trials would be invaluable in determining the "fit" elderly patient from the "average" elderly patients. While current clinical trials may identify the presence or absence of comorbidities, the severity of comorbidities should also be assessed. Without better characterization of the study population, our current knowledge on safety of TKIs on elderly patients may not be applicable to the "average" elderly patients.

In summary, we presented a case of an elderly patient with renal cell carcinoma (RCC) who developed multiple adverse events on tyrosine kinase inhibitors (TKIs). Our case highlights the many challenges that remain in treating elderly patients with RCC. As new prediction and assessment tools become available, how do we incorporate it into our management of elderly patients with cancer to identify those patients most atrisk for treatment adverse effects? In 2015, ASCO issued landmark recommendations calling for the need to broaden clinical trials to include older patients [33]. As the population ages and the number of elderly patients with cancer increases, this case illustrates the challenges of using TKIs in elderly patients as well as the growing need for more clinical trials that focus on elderly patients with cancer.

\section{References}

1. Bellmunt J, Négrier S, Escudier B, Awada A, Aapro M (2009) The medical treatment of metastatic renal cell cancer in the elderly: Position paper of a SIOG Taskforce. Crit Rev Oncol Hematol 69: 64-72.

2. Pal SK, Hsu J, Hsu S, Hu J, Bergerot P, et al. Impact of age on treatment trends and clinical outcome in patients with metastatic renal cell carcinoma. J Geriatr Oncol 4:128-133.

3. Nexavar (2013) Wayne, NJ: Bayer HealthCare Pharmaceuticals, Inc. and Emeryville, CA and Onyx Pharmaceuticals, Inc. South San Francisco, California, USA.

4. Eisen T, Oudard S, Szczylik C, Gravis G, Heinzer H, et al. (2008) Sorafenib for older patients with renal cell carcinoma: A subset analysis from a randomized trial. JNCI J Natl Cancer Inst 100: 1454-1463.

5. Bukowski RM, Stadler WM, McDermott DF, Dutcher JP, Knox JJ, et al. (2010) Safety and efficacy of sorafenib in elderly patients treated in the North American advanced renal cell carcinoma sorafenib expanded access program. Oncology 78: 340-347.

6. Procopio G, Bellmunt J, Dutcher J, Bracarda S, Knox J, et al. (2013) Sorafenib tolerability in elderly patients with advanced 
renal cell carcinoma: Results from a large pooled analysis. $\mathrm{Br} \mathrm{J}$ Cancer 108: 311-318.

7. EMA (2015) Sorafenib (Nexavar $\left.{ }^{\circledR}\right)$ - Summary of product characteristics.

8. Votrient (Pazopanib) (2014) Research Triangle Park, NC: GlaxoSmithKline, USA.

9. Sternberg CN, Davis ID, Mardiak J, Szczylik C, Lee E, et al. (2010) Pazopanib in locally advanced or metastatic renal cell carcinoma: Results of a randomized phase III trial. J Clin Oncol 28: 1061-1068.

10. EMA (2015) Pazopanib (Votrient $\left.{ }^{\circledR}\right)$-Summary of product characteristics.

11. Motzer RJ, Michaelson MD, Redman BG, Hudes GR, Wilding G, et al. (2006) Activity of SU11248, a multi-targeted inhibitor of vascular endothelial growth factor receptor and platelet-derived growth factor receptor, in patients with metastatic renal cell carcinoma. J Clin Oncol 24: 16-24.

12. Motzer RJ, Rini BI, Bukowski RM, Curti BD, George DJ, et al. (2016) Sunitinib in patients with metastatic renal cell carcinoma. JAMA 295: 2516-2524.

13. Sutent (sunitinib malate) Package Insert. Pfizer Inc. New York, USA.

14. Gore ME, Szczylik C, Porta C, Bracarda S, Bjarnason GA, et al. (2009) Safety and efficacy of sunitinib for metastatic renal-cell carcinoma: An expanded-access trial. Lancet Oncol 10: 757-763.

15. Van der Veldt AAM, Boven $E$, Helgason $H H$, Van Wouwe $M$, Berkhof J, et al. (2008) Predictive factors for severe toxicity of sunitinib in unselected patients with advanced renal cell cancer. Br J Cancer 99: 259-265.

16. Rini BI, Escudier B, Tomczak P, Kaprin A, Szczylik C, et al. (2011) Comparative effectiveness of axitinib versus sorafenib in advanced renal cell carcinoma (AXIS): a randomised phase 3 trial. Lancet 378: 1931-1939.

17. Access to Medicines (1998) A simple mHealth data platform to identify children that miss immunizations. Data on file, Pfizer, New York, USA.

18. Rini BI, Melichar B, Ueda T, Grünwald V, Fishman MN, et al. (2013) Axitinib with or without dose titration for first-line metastatic renal-cell carcinoma: A randomised double-blind phase 2 trial. Lancet Oncol 14: 1233-1242.

19. Mittal K, Wood LS, Rini BI (2012) Axitinib in metastatic renal cell carcinoma. Biol Ther 2: 5 .

20. Heath El, Infante J, Lewis LD, Luu T, Stephenson J, et al. (2013) A randomized, double-blind, placebo-controlled study to evaluate the effect of repeated oral doses of pazopanib on cardiac conduction in patients with solid tumors. Cancer Chemother Pharmacol 71: 565-573.
21. Dasanu CA, Padmanabhan P, Clark BA, Do C (2012) Cardiovascular toxicity associated with small molecule tyrosine kinase inhibitors currently in clinical use. Expert Opin Drug Saf 11: 445-457.

22. Escudier B, Porta C, Bono P, Powles T, Eisen T, et al. (2014) Randomized, controlled, double-blind, cross-over trial assessing treatment preference for pazopanib versus sunitinib in patients with metastatic renal cell carcinoma: PISCES Study. J Clin Oncol 32: 1412-1418.

23. Motzer RJ, Hutson TE, Cella D, Reeves J, Hawkins R, et al. (2013) Pazopanib versus sunitinib in metastatic renal-cell carcinoma. $\mathrm{N}$ Engl J Med 369: 722-731.

24. Hurria A (2009) Geriatric assessment in oncology practice. J Am Geriatr Soc 57: 246-249.

25. Extermann M, Hurria A (2007) Comprehensive geriatric assessment for older patients with cancer. J Clin Oncol 25: 1824-1831.

26. Aaldriks AA, Maartense E, Le Cessie S, Giltay EJ, Verlaan HACM, et al. (2011) Predictive value of geriatric assessment for patients older than 70 years, treated with chemotherapy. Crit Rev Oncol Hematol 79: 205-212.

27. Mohile SG, Bylow K, Dale W, Dignam J, Martin K, et al. (2007) A pilot study of the vulnerable elders survey-13 compared with the comprehensive geriatric assessment for identifying disability in older patients with prostate cancer who receive androgen ablation. Cancer 109: 802-810.

28. Overcash JA, Beckstead J, Moody L, Extermann M, Cobb S (2006) The abbreviated comprehensive geriatric assessment (aCGA) for use in the older cancer patient as a prescreen: Scoring and interpretation. Crit Rev Oncol Hematol 59: 205-210.

29. Luciani A, Ascione G, Bertuzzi C, Marussi D, Codecà C, et al. (2010) Detecting disabilities in older patients with cancer: Comparison between comprehensive geriatric assessment and vulnerable elders survey-13. J Clin Oncol 28: 2046-2050.

30. Bellera CA, Rainfray M, Mathoulin-Pélissier S, Mertens C, Delva F, et al. (2012) Screening older cancer patients: First evaluation of the G-8 geriatric screening tool. Ann Oncol 23: 2166-2172.

31. Hurria A, Togawa K, Mohile SG, Owusu C, Klepin HD, et al. (2011) Predicting chemotherapy toxicity in older adults with cancer: $A$ prospective multicenter study. J Clin Oncol 29: 3457-3465.

32. Extermann $M$, Boler I, Reich RR, Lyman GH, Brown RH, et al. (2012) Predicting the risk of chemotherapy toxicity in older patients: The Chemotherapy Risk Assessment Scale for High-Age Patients (CRASH) score. Cancer 118: 3377-3386.

33. Hurria A, Levit LA, Dale W, Mohile SG, Muss HB, et al. (2015) Improving the evidence base for treating older adults with cancer: American Society of Clinical Oncology Statement. J Clin Oncol 32: 3826-3833. 